\title{
COMPARATIVE EFFECTS OF A PURIFIED AND AN ADEQUATE DIET ON THE COURSE OF FATTY CIRRHOSIS IN THE ALCOHOLIC ${ }^{1}$
}

\author{
By GERALD B. PHILLIPS, GEORGE J. GABUZDA, JR.,² AND CHARLES S. DAVIDSON \\ (From the Thorndike Memorial Laboratory, Second and Fourth Medical Services [Harvard], \\ Boston City Hospital, and the Department of Medicine, Harvard Medical School, \\ Boston, Mass.)
}

(Submitted for publication November 5, 1951; accepted January 21, 1952)

The association of alcoholism and cirrhosis of the liver is widely recognized; nevertheless, it is still not clear whether the hepatic disturbance is the result of (1) an hepatotoxic effect of the alcohol or a contaminant it may contain, (2) a nutritional deficiency, or (3) a combination of the two. The concept that cirrhosis in the alcoholic may be a deficiency disease has gained popularity ever since the demonstration that fatty cirrhosis similar to that seen in man could be produced in animals by means of low protein diets $(1,2)$. In support of this view is the association of fatty cirrhosis with states of malnutrition unaccompanied by alcoholism $(3,4)$. Moreover, the apparent therapeutic efficacy of a nutritious diet, as reported by Patek and Post (5, $6)$, further suggests nutritional deficiency as an etiologic factor. But whether the success of this regimen resides in the diet, withdrawal of alcohol, bed rest, or in a combination of these factors, is as yet unsettled.

Recently, Eckhardt and his associates (7) followed three chronic alcoholics with fatty cirrhosis maintained for eight to 13 days on a diet essentially devoid of protein but adequate in calories and supplemented with a multivitamin preparation. ${ }^{3}$ These patients showed striking improvement in clinical status and in hepatic function tests within a week, although histologically liver fat did not decrease significantly except in one patient during a period of choline supplementation.

1 The expenses of this investigation were defrayed in part by grants from Merck \& Co., Inc., Rahway, New Jersey, and from the Commission on Liver Disease, Armed Forces Epidemiological Board, Office of the Surgeon General, U. S. Army, Washington, D. C., to Harvard University.

2 Welch Fellow in Internal Medicine of the National Research Council.

3 This diet maintained nitrogen balance and body weight in a normal adult male when casein was provided orally as the sole source of protein.
To elucidate further the role of diet, three chronic alcoholics with large fatty livers were maintained on a highly purified diet. After eight to ten days of this regimen, an adequate diet was provided and the study continued for an additional eight to 19 days. Liver function, size, and histology were evaluated at appropriate intervals.

\section{MATERIALS AND METHODS}

Brief descriptions of the three patients included in this study follow.

Patient B. R. was a 56-year-old white male, chronic alcoholic who had been drinking heavily and eating meagerly for three weeks prior to admission. On entry, a firm tender liver edge was felt $4 \mathrm{~cm}$. below the costal margin in the midclavicular line, but no edema, ascites, icterus, splenomegaly or spider telangiectasia was detected. Because he was thoroughly confused and manifested a marked tremor, severe ataxia, coarse nystagmus, and bilateral external rectus muscle paralysis, the diagnosis of Wernicke's syndrome with probable delirium tremens was made.

Patient P. F. was a 46-year-old white male who had been drinking steadily for 20 to 30 years and had had frequent episodes suggestive of delirium tremens. Nine months before the present admission, he had been hospitalized because of jaundice and was found to have hepatomegaly with marked functional impairment. Since that time he had been imbibing large quantities of alcoholic beverages and eating little. On admission, his liver edge was $10 \mathrm{~cm}$. below the costal margin in the midclavicular line, firm, and non-tender. There was no evidence of edema, ascites, icterus, splenomegaly, or spider telangiectasia. He exhibited marked confusion, ataxia and bilateral external rectus weakness and was considered to have Wernicke's syndrome.

Patient R. W. was a 28-year-old white female who had been an alcoholic for about six years and had been drinking heavily and steadily for the last four to five years. She admitted to consuming only small quantities of food, often eating nothing for three to four days at a time. Icteric sclerae were noted several weeks prior to entry. No definite history of previous hepatic disturbance could be elicited. Physical examination on admission revealed scleral icterus, palmar erythema, numerous prominent 
spicler telangiectases, and a large tender liver, which extended almost to the iliac crest. Ascites and edema were not evident.

During the initial period of hospitalization these patients were given a purified diet which consisted of a solution containing $200 \mathrm{gm}$. of glucose and $1.3 \mathrm{gm}$. of sodium chloride per liter of water. B. R. and P. F. consumed approximately 2 liters of this solution daily (i.c., about 1,600 calories and $2.6 \mathrm{gm}$. sodium chloride), while R. W., who frequently had nausea and vomiting following ingestion of the solution, was able to retain only about a liter (i.e., about 800 calories and $1.3 \mathrm{gm}$. sodium chloride) a day. In addition, $\mathrm{R}$. W. ingested $6 \mathrm{gm}$. of potassium chloride a day during the period of the purified diet. None of the patients received any sustenance after entering the hospital except for the glucose and minerals, which were begun within a few hours after admission and continued for eight days in the case of patients P. F. and R. W. and 10 days in the case of patient $\mathrm{B}$. $\mathrm{R}$. On cessation of the purified diet, an adequate diet was provided for the remainder of the patient's hospital stay. The adequate diet consisted of the unsupplemented regular hospital diet $(2,000$ to 2,200 calories-protein 80 to $100 \mathrm{gm}$., carbohydrate 200 to $250 \mathrm{gm}$., and fat approximately $110 \mathrm{gm}$. daily). An accurate measure was not made of the quantity of food ingested by each patient. B. R. and P. F. ate moderately well during the first week of the adequate diet, while R. W. consumed only a small fraction of what was offered to her, frequently refusing entire meals. After the first week of this diet, R. W.'s appetite and food intake gradually increased. No medication was administered after admission other than small doses of paraldehyde for B. R. and $P$. F. and penicillin during three days to $P$. F. because of cough without fever, increased chest markings by X-ray, and an elevated white blood count.

All patients were ambulatory after the second or third day in the hospital.

Because patients B. R. and P. F. had definite evidence of Wernicke's syndrome, thiamine was administered to both with subsequent improvement in their neurological abnormalities. ${ }^{4}$

Liver function, size, and histology were evaluated on the day of admission and at appropriate times during the study. To appraise hepatic function, the following measurements were made: bromsulphalein retention 45 minutes after the intravenous administration of the dye $(5 \mathrm{mg}$. per $\mathrm{Kg}$. of body weight calculated on the basis of initial body

* B. R. received $100 \mathrm{mg}$. of thiamine hydrochloride subcutaneously twice a day from days 4 to 10 , while P. F. was given $5 \mathrm{mg}$. intravenously on day $2,15 \mathrm{mg}$. orally from days 3 to 6 , and $50 \mathrm{mg}$. subcutaneously on days 7 and 8 . In addition, $B$. $R$. received $1 \mathrm{gm}$. of niacin orally from days 7 to 10 and $4 \mathrm{cc}$. of an intravenous multi-vitamin preparation, "Berocca-C" (Hoffmann-LaRoche), on days 8 and 9 . Each $2 \mathrm{cc}$. of this contains thiamine hydrochloride, $10 \mathrm{mg}$.; riboflavin, $6 \mathrm{mg}$.; niacinamide, $80 \mathrm{mg}$.; pyridoxine hydrochloride, $6 \mathrm{mg}$; panthenol (equivalent to $6 \mathrm{mg}$. Ca pantothenate), $6 \mathrm{mg}$.; ascorbic acid, $100 \mathrm{mg}$.; with $3 \%$ gentisic acid ethanolamide as a preservative.
TABLE I

Patient B. R., male, age 56

\begin{tabular}{|c|c|c|c|c|c|c|}
\hline \multicolumn{2}{|l|}{ Day of study } & 0 & 5 & 8 & 10 & \multirow{2}{*}{$\frac{18}{\begin{array}{c}\text { Adequate } \\
\text { diet }\end{array}}$} \\
\hline Intake & & \multicolumn{4}{|c|}{ Glucose and saline* } & \\
\hline \multicolumn{2}{|c|}{ Bromsulphalein, \% retention } & 41 & 51 & 43 & 61 & 32 \\
\hline \multirow{2}{*}{$\begin{array}{l}\text { Serum bilirubin } \\
m g . / 100 \mathrm{cc} .\end{array}$} & "1 min." & 0.92 & 0.76 & 0.63 & 0.31 & 0.27 \\
\hline & Total & 2.3 & 1.8 & 1.7 & 1.2 & 0.74 \\
\hline \multicolumn{2}{|l|}{$\begin{array}{l}\text { Thymol turbidity† } \\
c c . \mathrm{BaSO}_{4}\end{array}$} & & 0.7 & 0.6 & 0.7 & 1.3 \\
\hline \multicolumn{2}{|c|}{ Thymol flocculation $(0-4+)$} & & 0 & 0 & 0 & 0 \\
\hline \multicolumn{2}{|l|}{ Urine urobilinogen } & $1: 256$ & & $1: 256$ & $1: 256$ & $1: 64$ \\
\hline \multicolumn{2}{|l|}{ Urine bile $(0-4+)$} & & & $2+$ & $2+$ & $\mathbf{0}$ \\
\hline \multicolumn{2}{|l|}{ Liver size $\ddagger$} & $4 \mathrm{~cm}$ & & $5 \mathrm{~cm}$ & & \\
\hline \multicolumn{2}{|l|}{ Hepatic fat $(0-4+)$} & $4+$ & & $3+$ & $4+$ & $2+$ \\
\hline
\end{tabular}

* Patient received $100 \mathrm{mg}$. of thiamine hydrochloride subcutaneously twice a day from days 4 to $10,1 \mathrm{gm}$. of niacin orally from days 7 to 10 , and $4 \mathrm{cc}$. of an intravenous multivitamin preparation ("Berocca-C") on days 8 and 9.

$\dagger$ Normal value $-<1.7$ cc. $\mathrm{BaSO}_{4}$ suspension.

$\ddagger$ Below costal margin in midclavicular line.

weight), "one minute" and total serum bilirubin (8), thymol turbidity and flocculation (9), urine urobilinogen (10) and bile (11), and plasma prothrombin concentration (12).

Punch biopsies of the liver were performed using the Vim-Silverman needle. In patient R. W. a subcostal anterior approach was employed, while in the other two patients, the needle was inserted transthoracically in the lower axilla. For serial biopsies, an attempt was made to insert the needle at the site of the previous puncture. However, a shift in the biopsy site may have taken place

TABLE II

Patient P. F., male, age 46

\begin{tabular}{|c|c|c|c|c|c|c|}
\hline \multicolumn{2}{|l|}{ Day of study } & $\mathbf{0}$ & 6 & 8 & 16 & 22 \\
\hline \multicolumn{2}{|l|}{ Intake } & \multicolumn{3}{|c|}{ Glucose and saline* } & \multicolumn{2}{|c|}{ Adequate diet } \\
\hline \multicolumn{2}{|c|}{ Bromsulphalein, \% retention } & 37 & 40 & 45 & 31 & 26 \\
\hline \multirow{2}{*}{$\begin{array}{l}\text { Serum bilirubin } \\
m g . / 100 c c\end{array}$} & "1 min." & 1.2 & 1.4 & 1.9 & 0.59 & 0.48 \\
\hline & Total & 2.1 & 2.9 & 3.4 & 1.2 & 1.0 \\
\hline \multicolumn{2}{|l|}{$\begin{array}{l}\text { Thymol turbidity } \\
\text { cc. } \mathrm{BaSO}_{4}\end{array}$} & 4.1 & 3.6 & 4.9 & 3.3 & 3.6 \\
\hline \multicolumn{2}{|c|}{ Thymol flocculation $(0-4+)$} & $1+$ & $1+$ & $1+$ & $1+$ & $2+$ \\
\hline \multicolumn{2}{|l|}{ Urine urobilinogen } & $1: 256$ & $1: 512$ & $1: 512$ & $1: 256$ & $1: 128$ \\
\hline \multicolumn{2}{|l|}{ Urine bile $(0-4+)$} & $2+$ & $2+$ & $2+$ & $2+$ & $1+$ \\
\hline \multicolumn{2}{|l|}{ Liver size } & $10 \mathrm{~cm}$ & & $10 \mathrm{~cm}$. & $9 \mathrm{~cm}$ & \\
\hline \multicolumn{2}{|l|}{ Hepatic fat $(0-4+)$} & $4+$ & & $4+$ & $3+$ & $2+$ \\
\hline
\end{tabular}

* Patient received $5 \mathrm{mg}$. thiamine hydrochloride intravenously on day $2,15 \mathrm{mg}$. orally from days 3 to 6 , and $50 \mathrm{mg}$. subcutaneously on days 7 and 8 .

$\dagger$ Normal value $-<1.7 \mathrm{cc}$. $\mathrm{BaSO}_{4}$ suspension.

$\ddagger$ Below costal margin in midclavicular line. 
TABLE III

Patient R. W., female, age 28

\begin{tabular}{|c|c|c|c|c|c|c|c|c|}
\hline \multirow{2}{*}{\multicolumn{2}{|c|}{$\frac{\text { Day of study }}{\text { Intake }}$}} & $\mathbf{0}$ & 4 & 8 & 15 & 18 & 20 & 27 \\
\hline & & \multicolumn{3}{|c|}{ Glucose and saline* } & \multicolumn{4}{|c|}{ Adequate diet } \\
\hline \multicolumn{2}{|c|}{ Bromsulphalein, $\%$ retention } & 33 & 35 & 39 & 30 & 31 & 31 & 27 \\
\hline \multirow{2}{*}{$\begin{array}{l}\text { Serum bilirubin } \\
m g . / 100 c c\end{array}$} & “1 min." & 1.2 & 1.1 & 1.0 & 0.52 & 0.48 & 0.56 & 0.48 \\
\hline & Total & 2.5 & 2.5 & 2.2 & 1.3 & 1.0 & 1.0 & 0.99 \\
\hline \multicolumn{2}{|l|}{$\begin{array}{l}\text { Thymol turbidity } \dagger \\
\text { cc. } \mathrm{BaSO}_{4}\end{array}$} & 4.0 & 3.9 & 3.9 & 2.7 & 3.1 & 3.1 & 3.7 \\
\hline \multicolumn{2}{|c|}{ Thymol flocculation $(0-4+)$} & $3+$ & $3+$ & $3+$ & $1+$ & $1+$ & $1+$ & $1+$ \\
\hline \multicolumn{2}{|l|}{ Urine urobilinogen } & $1: 32$ & $1: 32$ & $1: 32$ & $1: 32$ & $1: 32$ & & $1: 32$ \\
\hline \multicolumn{2}{|l|}{ Urine bile $(0-4+)$} & 0 & 0 & 0 & 0 & 0 & & \\
\hline \multicolumn{2}{|l|}{ Liver size, $\mathrm{cm} . \ddagger$} & 15 & 15 & 15 & 13 & 13 & & 9 \\
\hline \multicolumn{2}{|l|}{ Hepatic fat $(0-4+)$} & $2+$ & & $2+$ & & 0 & & \\
\hline
\end{tabular}

* Patient received $6 \mathrm{gm}$. of potassium chloride daily from days 0 to 8 .

$\dagger$ Normal value $-<1.7 \mathrm{cc}$. $\mathrm{BaSO}_{4}$ suspension.

$\ddagger$ Below costal margin in midclavicular line.

due to changes in liver size, position of the patient, or phase of respiration. No untoward reactions occurred except in R. W., who developed a small tender mass, probably a hematoma, on the anterior surface of the liver after the first biopsy and experienced pain for one to two days after each subsequent one. The liver tissue was fixed in 10 per cent formalin, sectioned, and stained with hematoxylin and eosin. No other stains were used. In addition to the usual histological examination, fat, identified as large unstained vacuoles, was estimated on a $1+$ to $4+$ scale. ${ }^{j}$

\section{RESULTS}

The effects of the purified and adequate diets on hepatic function, size, and fat content are summarized in Tables I-III. The results obtained in the three patients studied are similar. During the purified diet period there was no evidence of hepatic functional improvement except for a diminution in serum bilirubin in two patients (B. R. and R. W.). The serum bilirubin increased in the third patient (P. F.). The impaired ability to excrete bromsulphalein exhibited by all three patients on admission was augmented still further during the period of the purified diet. Thymol turbidity

5 The authors are grateful to Dr. Frederic Parker, the Mallory Institute of Pathology, Boston City Hospital, for reviewing the histological sections and to Miss Lillian Leavitt of the same institution for preparing the biopsy specimens. and urine urobilinogen and bile either remained the same or became more abnormal. In no patient was there any detectable decrease in hepatic size.

An estimation of fat content and of the extent of fibrosis was made by microscopic examination of the stained sections of the liver biopsies (Figures 1-3). This method is at most semi-quantitative. In addition, the possibility exists that there is variation in these elements in different parts of the liver. B. R.'s initial liver biopsy showed marked fatty infiltration and minimal, if any, fibrosis (Figure 1). P. F., likewise, had a large amount of hepatic fat and in this instance definite fibrosis (Figure 2). R. W. had a moderate amount of hepatic fat but a considerable degree of fibrosis (Figure 3). The biopsies secured after eight days of the purified diet showed a slight decrease in hepatic fat in B. R. but no significant change in P. F. or R. W. A repeat biopsy performed on B. R. after ten days of the purified diet, however, disclosed a quantity of hepatic fat similar to that seen in the initial biopsy. Thus, a significant decrease in hepatic fat was not evidenced by any of the patients at the conclusion of the period of the purified diet.

Following the purified diet, the patients were given the adequate diet for the remainder of their hospitalization. Hepatic function was evaluated 

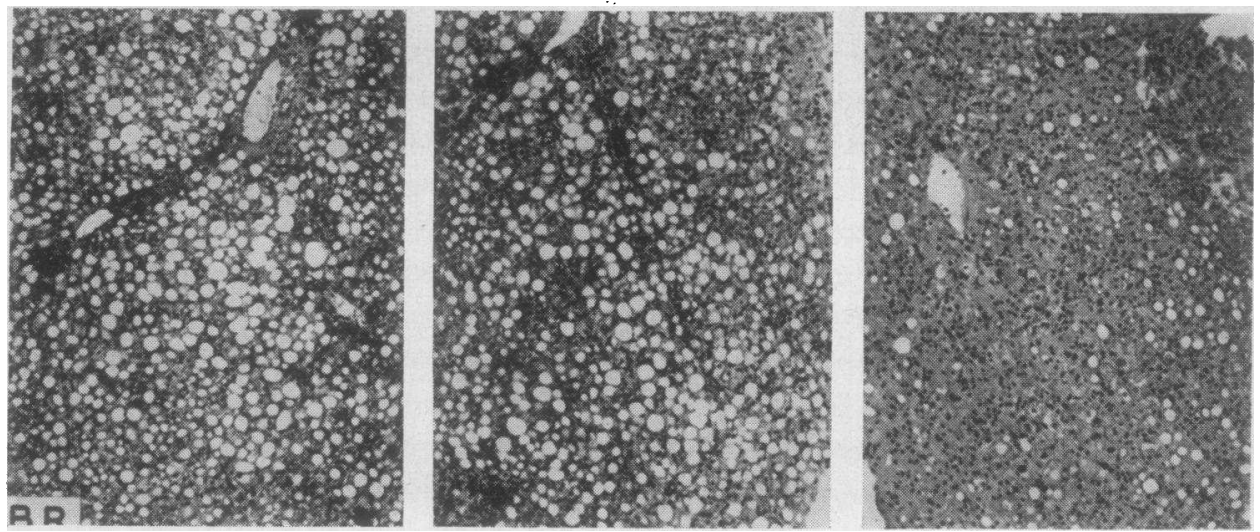

Fig. 1. Patient B. R.

The biopsies are in chronological sequence from left to right. The first biopsy was performed on admission, the second after ten days of the purified diet, and the third after eight days of the adequate diet. Hematoxylin and eosin $\times 50$.
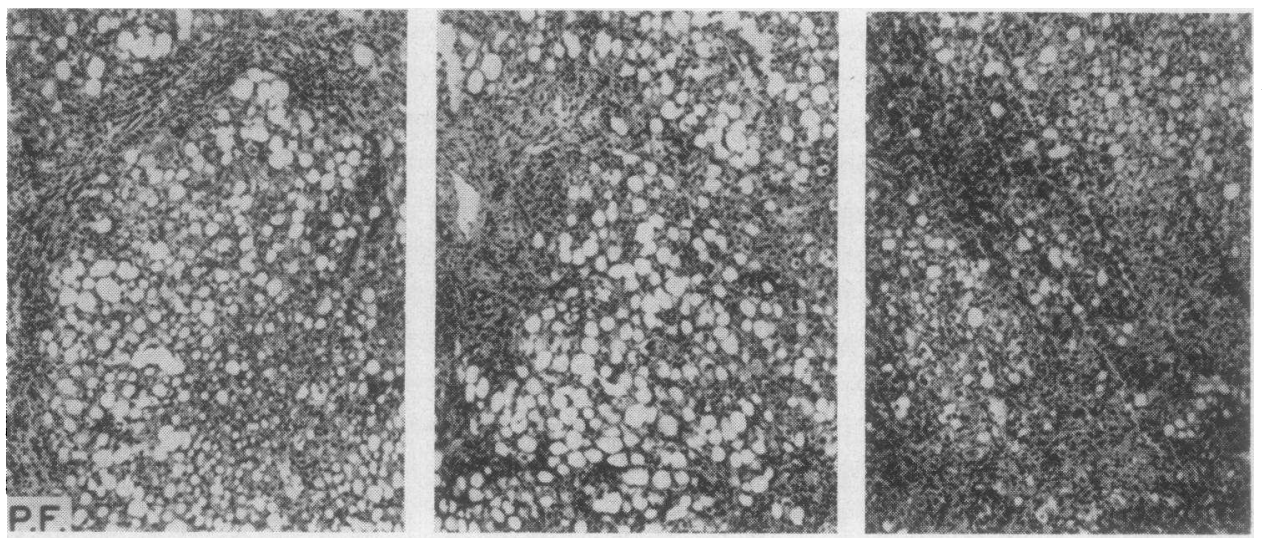

Fig. 2. Patient P. F.

The biopsies are in chronological sequence from left to right. The first biopsy was performed on admission, the second after eight days of the purified diet, and the third after 14 days of the adequate diet. Hematoxylin and eosin $\times 50$.
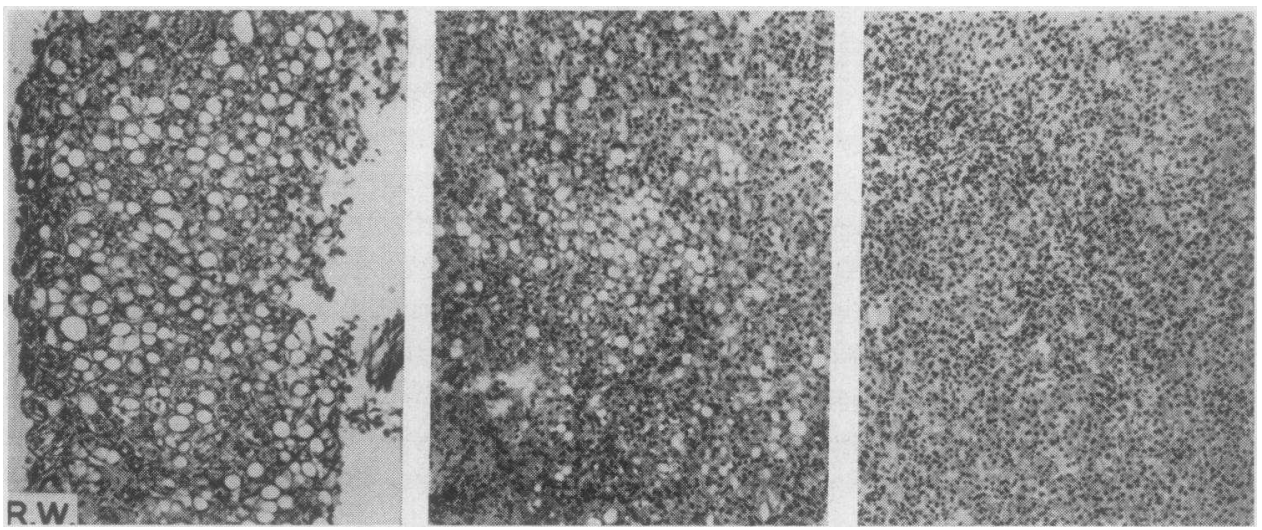

Fig. 3. Patient R. W.

The biopsies are in chronological sequence from left to right. The first biopsy was performed on admission, the second after eight days of the purified diet and the third after ten days of the adequate diet. Hematoxylin and $\operatorname{eosin} \times 50$. 
after eight days of this diet in B. R. and P. F., and after seven days in $R$. W. Considerable improvement was apparent by the end of this period in all three patients. Both the B. S. P. retention and serum bilirubin concentration decreased significantly, and the urine urobilinogens which were abnormal also returned toward normal.

Liver size decreased definitely in R. W. and possibly in P. F. It was not remeasured in B. R. There was a marked diminution in hepatic fat in B. R. and R. W. after eight and ten days, respectively, of the adequate diet, but the decrease was slight, if any, in P. F. after eight days. A repeat biopsy in P. F. after 14 days of the adequate diet, however, revealed a definite decrease in hepatic fat.

\section{DISCUSSION}

That most chronic alcoholics with fatty cirrhosis improve rapidly when hospitalized and provided with a regular hospital diet has been the experience of this clinic as well as of others (13-17). But the relative importance of food, alcohol withdrawal and bed rest in this rapid recovery has not been clarified sufficiently. Although fatty cirrhosis can be produced in animals by means of low protein diets and prevented by methionine or choline, Eckhardt and his coworkers (7), in a previous study from this laboratory, observed that dramatic improvement in clinical status and hepatic function without significant decrease in hepatic fat occurred in three chronic alcoholics with fatty cirrhosis studied when they were maintained on a diet containing essentially no protein. Their diet did contain, however, an estimated 30 to $100 \mathrm{mg}$. of choline and one vitamin capsule ${ }^{6}$ per day.

In the present study, three similar patients were furnished with a more highly purified diet, consisting only of a solution of glucose and minerals, and studied under comparable conditions and for approximately the same length of time. Unlike the results obtained by Eckhardt and his associates, no significant improvement in hepatic function or size occurred. In fact, measurements of hepatic function became decidedly worse in one patient.

\footnotetext{
- For vitamins furnished in the present study, see Footnote 4 ; Eckhardt and his coworkers gave one "Dayamin" capsule daily containing thiamine hydrochloride, $5 \mathrm{mg}$.; riboflavin, $5 \mathrm{mg}$.; nicotinamide, $25 \mathrm{mg}$.; pantothenic acid, $5 \mathrm{mg}$.; pyridoxine, $1.5 \mathrm{mg}$.; ascorbic acid, $100 \mathrm{mg}$.; vitamin A, 10,000 U.S.P. units ; and vitamin D, 1,000 U.S.P. units.
}

Similar to their findings however, no change in hepatic fat content was apparent. In a second period of study of similar length, all conditions were kept constant except for a change from the purified to an adequate diet. The rapid improvement in hepatic function was in sharp contrast to the lack of improvement in the initial period of study. In addition, there was a considerable decrease in hepatic fat in two of the three patients, but this decrease was not apparent in the third patient until two weeks had elapsed.

It should be noted that the purified diet of the patients in this study differed not only qualitatively from the diet used by Eckhardt and his coworkers (7) but also quantitatively, as the former provided 1,600 calories or less per day, while the latter supplied almost 3,000 calories per day. R. W. consumed only about 14 calories/Kg./day, but P. F. and B. R. were able to take approximately 21 and 27 calories $/ \mathrm{Kg}$./day, respectively. At least in the latter two patients, this caloric intake should be sufficient for a normal sedentary person. Moreover, while R. W.'s caloric intake was low on the purified diet, it was also low on the adequate diet, although precise measurements of the latter were not made. The possibility remains that the low caloric value of the purified diet might have contributed to the failure of these patients to recover.

Because of co-existing Wernicke's syndrome, two of the patients received thiamine in addition to the purified diet with satisfactory amelioration of the abnormal neurological signs. However, no beneficial effect on the hepatic process could be attributed to the thiamine. The short term use of multivitamins in one patient was associated with a rise in B.S.P. retention and an apparent increase in hepatic fat; this single observation, however, does not justify interpretation.

The results observed attest to the efficacy of the adequate diet or of one or more of its constituents in the successful treatment of the fatty cirrhosis in these patients and suggest dietary deficiency as an important etiologic factor. The failure of these patients to improve when provided with a highly purified diet, in spite of alcohol withdrawal and rest, indicates that these factors probably played subsidiary roles, especially since improvement did occur when an adequate diet was substituted. The therapeutic component or components of an adequate diet await positive identification for man. 


\section{SUM MARY}

The effects of a highly purified and subsequently of an adequate diet on hepatic function, size, and fat content were studied in three chronic alcoholics with fatty cirrhosis of the liver.

None of the three patients showed significant improvement in hepatic function, size, or fat content during the eight to ten days of a purified diet, consisting of glucose and minerals with the addition of thiamine alone in one patient and thiamine plus multivitamins in another. These results are in contrast to those obtained in a previous study in this laboratory in which three similar patients showed improvement in hepatic function and size while on a less purified diet.

After institution of an adequate diet for eight to ten days, there was improvement in hepatic function in all three patients with a decrease in hepatic fat in two. The third patient showed a significant decrease in fat in the biopsy taken after 14 days of the adequate diet.

These results suggest that the improvement observed was related to the provision of an adequate diet and that a subsidiary, if any, role was played by withdrawal of alcohol and rest in the hospital.

\section{REFERENCES}

1. György, P., The nutritional aspects of liver injury. M. Clin. North America, 1949, 33, 1657.

2. Himsworth, H. P., Lectures on the Liver and its Diseases. Harvard University Press, Cambridge, 1950.

3. Davidson, C. S., and Gabuzda, G. J., Jr., Nutrition and disease of the liver. New England J. Med., 1950, 243, 779.

4. Gillman, J., and Gillman, T., Perspectives in Human Malnutrition. Grune \& Stratton, Inc., New York, 1951.

5. Patek, A. J., Jr., Treatment of alcoholic cirrhosis of the liver with high vitamin therapy. Proc. Soc. Exper. Biol. \& Med., 1937, 37, 329.
6. Patek, A. J., Jr., and Post, J., Treatment of cirrhosis of the liver by a nutritious diet and supplements rich in vitamin B complex. J. Clin. Invest., 1941, 20, 481.

7. Eckhardt, R. D., Zamcheck, N., Sidman, R. L., Gabuzda, G. J., Jr., and Davidson, C. S., The effect of protein starvation and of protein feeding on the clinical course, liver function and liver histochemistry of three patients with active fatty alcoholic cirrhosis. J. Clin. Invest., 1950, 29, 227.

8. Ducci, H., and Watson, C. J., The quantitative determination of the serum bilirubin with special reference to the prompt-reacting and chloroformsoluble types. J. Lab. \& Clin. Med., 1945, 30, 293.

9. Ley, A. B., Lewis, J. H., and Davidson, C. S., The quantitative determination of the thymol turbidity reaction of serum. J. Lab. \& Clin. Med., 1946, 31, 910.

10. Wallace, G. B., and Diamond, J. S., The significance of urobilinogen in the urine as a test for liver function. With a description of a simple quantitative method for its estimation. Arch. Int. Med., 1925, 35, 698 .

11. Harrison, G. A., Chemical Methods in Clinical Medicine: Their Application and Interpretation with the Technique of the Simple Tests. J. \& A. Churchill, London, 1937, 2nd Ed.

12. Souter, A. W., and Kark, R., Quick's prothrombin test simplified by the use of a stable thromboplastin. Am. J. M. Sc., 1940, 200, 603.

13. Chalmers, T. C., Murphy, T. L., and Taft, E. B., The incidence, character, and course of liver disease in chronic alcoholics as determined by needle biopsy. J. Clin. Invest., 1948, 27, 528.

14. Klatskin, G., and Yesner, R., Factors in the treatment of Laennec's cirrhosis. I. Clinical and histological changes observed during a control period of bedrest, alcohol withdrawal, and a minimal basic diet. $\mathrm{J}$. Clin. Invest., 1949, 28, 723.

15. Volwiler, W., Jones, C. M., and Mallory, T. B., Criteria for the measurement of results of treatment of fatty cirrhosis. Gastroenterology, 1948, 11, 164.

16. Buck, R. E., Observations on alcoholic fatty liver: The use of interval needle biopsy and liver function tests. J. Lab. \& Clin. Med., 1948, 33, 555.

17. Seife, M., Kessler, B. J., and Lisa, J. R., Clinical, functional, and needle biopsy study of the liver in alcoholism. Arch. Int. Med., 1950, 86, 658. 